\title{
POST-CRITICAL DEFORMATION OF THIN-WALLED LOAD-BEARING AIRCRAFT STRUCTURE REPRESENTING FRAGMENT OF THE ONE-WAY TORSION BOX
}

\author{
Tomasz Kopecki ${ }^{1}$, Tomasz Lis ${ }^{1}$, Przemysław Mazurek \\ 1 Rzeszów University of Technology, The Faculty of Mechanical Engineering and Aeronautiucs, Al. Powstańców \\ Warszawy 8, 35-959 Rzeszów, Poland. e-mail: tkopecki@prz.edu.pl, list@prz.edu.pl, pmazurek@prz.edu.pl
}

Received: 2018.06.28

Accepted: 2018.08.05

Published: 2018.09.01

\begin{abstract}
The paper presents results of research on thin-walled load-bearing structure model representing a fragment of plane wing torsion box. The basis for obtaining data on deformation character and stability loss process was a model experiment conducted using a dedicated experimental stand. On the basis of experiment results, adequate numerical simulations were conducted using software based on finite elements method. Results of non-linear numerical analyses allowed to determine the character of stress distribution and formulate conclusions regarding behaviour of the post-critical deformations of the examined wing part.
\end{abstract}

Keywords: buckling, finite elements method, nonlinear analysis, polycarbonate, experimental stand, stress distributions.

\section{INTRODUCTION}

Despite several innovative solutions, the creation of which is characteristic foe the development of current aircraft technology, significant majority of designed airframes utilises typical load-bearing structures elements based on already tested structural diagrams [11]. One of these commonly used solutions is utilising a wing torsion box ensuring correct torsional rigidity and resistance to torsion of the structure. Wing torsion boxis usually a thin-walled circuit of external cross-section contour determined by geometry of the utilised aerodynamic profile and wing spar elements surfaces (Fig. 1).

Due to numerous exploitative conditions, in majority of cases the first of the aforementioned solution is used, in which torsion box circuitis placed in the forward part of the wing profile and is limited by a wing spar wall, placed usually in the area of maximum relative thickness of the profile.

For years, aircraft structure design has followed the rule thatcovering fragments placed out- side torsion box area can undergo stability loss if it occurs locally, only in the area of covering segments limited with frame elements, and is elastic in character $[8,10,12]$.

Regarding the area of torsion box, however, there is a different constructional doctrine. In this case, stability loss is connected to the rapid decrease of torsional rigidity of a wing and changes in its geometry causing decrease of its aerodynamic properties. Thus, torsion box wall thickness is selected primarily on the basis of its stability requirement $[1,7]$.

Even though the torsion box covering buckling is not admissible in exploitative stress conditions, during the design process stress increases resulting from a momentary atmosphere state and performed manoeuvres, which my locally exceed critical values, are considered as well. In such cases, it should be assumed that the stability loss may be rapid and present risk of damage or destruction to the wing load-bearing structure [16]. Therefore, introducing structural solutions securing the loadbearing structure from effects of such rapid occurrences should be considered, and a detailed analy- 

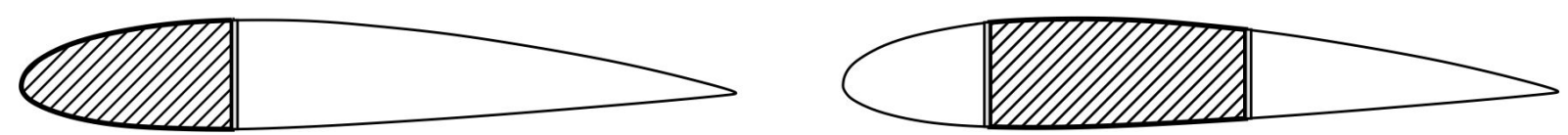

Fig. 1. Examples of wing caisson constructional diagrams

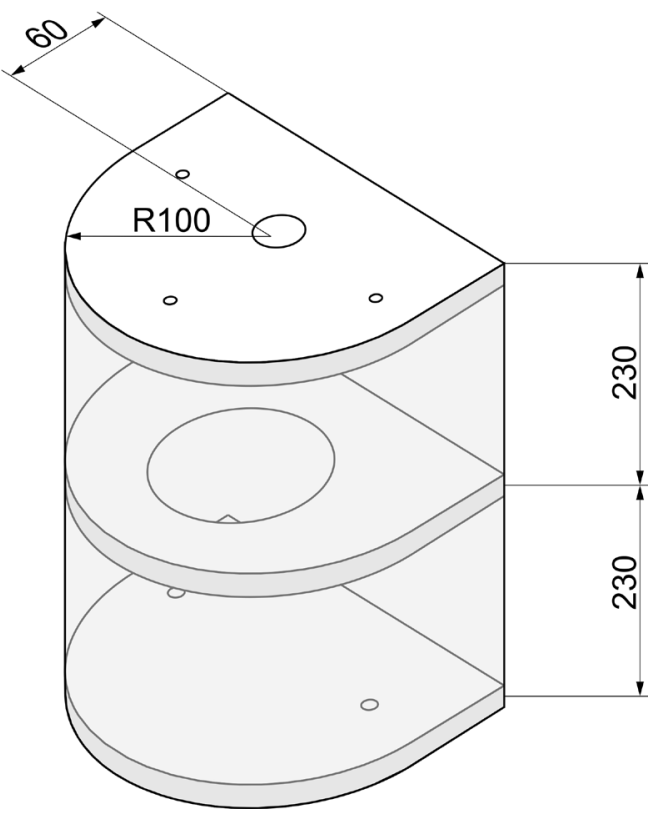

Fig. 2. Structural diagram

sis of torsion box covering deformation and stress distribution in conditions of post-critical stress should be conducted $[5,6,13,15]$.

\section{RESEARCH RANGE AND OBJECTIVE}

The subject of the research was a fragment of thin-walled load-bearing structure corresponding to a torsion box part of plane wing (Fig. 2) undergoing torsion.

Model for the experimental tests was made of polycarbonate of commercial name "macrolon", for which, as a result of a conducted tensile test, the following material features were determined: Young module $\mathrm{E}=3000 \mathrm{MPa}$ and Poisson ratio $v=0.36$.

Ribs thickness equalled $10 \mathrm{~mm}$, covering thickness $-0.7 \mathrm{~mm}$. Holes were drilled in upper and lower rib to enable mounting the model on the experimental stand and applying load.

Characteristics of the aforementioned material during one-dimensional tensile test were determined as well (Fig. 3).

Although a presence of separate elastic and non-elastic deformation areas allows to assume

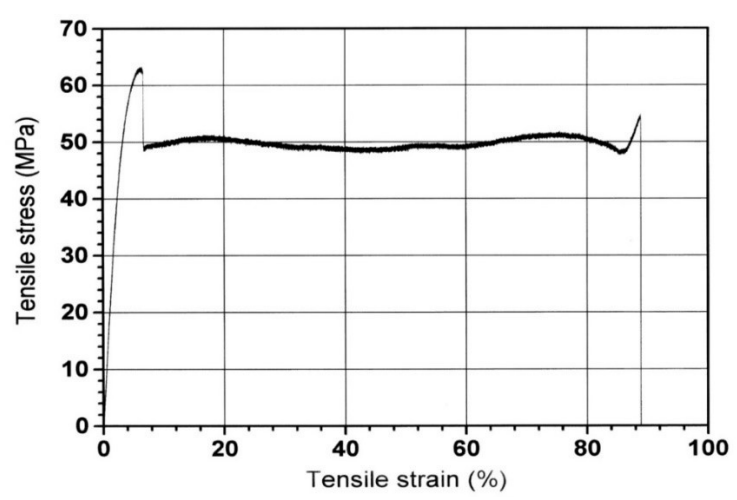

Fig. 3. Characteristics of $\sigma-\varepsilon$ for one-dimensional tensile test

an ideally elastic and plastic material model, for the numerical calculations an ideally elastic model was used. It is a result of limits enforced by laws of aircraft construction, which allows for only elastic character of structure deformation.

The polycarbonate is a material of high optical sensitivity, which enabled qualitative determining of stress distribution using photo elasticity methods. For that purpose, covers interior surfaces were covered with reflective layers.

Experimental tests results were a basis of specifying a numerical model, conducted using MSC PATRAN software based on the finite elements method. Nonlinear numerical analyses were conducted using MSC MARC software.

\section{EXPERIMENTAL TESTS}

Experimental tests were conducted using a dedicated experimental stand (Fig. 4a). The load was applied gravitationally (Fig. 5).

Measurements of stressing system elements displacement were conducted during the experiment using micrometric sensors, due to which it was possible to determine total torsion angle of the structure for each tested load state. As a result, a representative equilibrium path was received, which is a relation between the torsion moment and the total torsion angle (Fig. 6). Moreover, for advanced structure deformation states, their numerical representation was created using optical scanner (Fig. 7). 

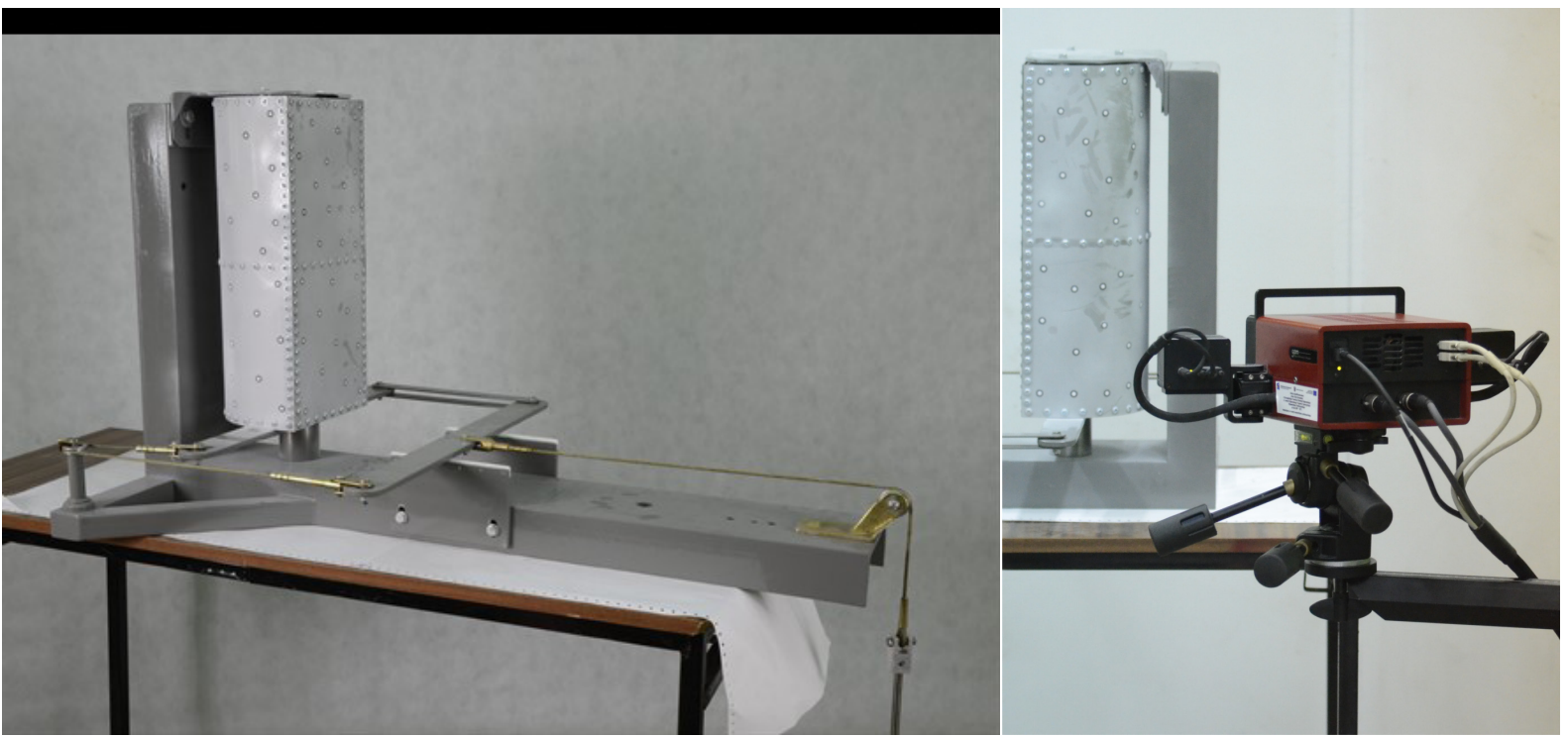

Fig. 4. a) The experimental stand with installed model, b) Deformation measurements using optical scanner

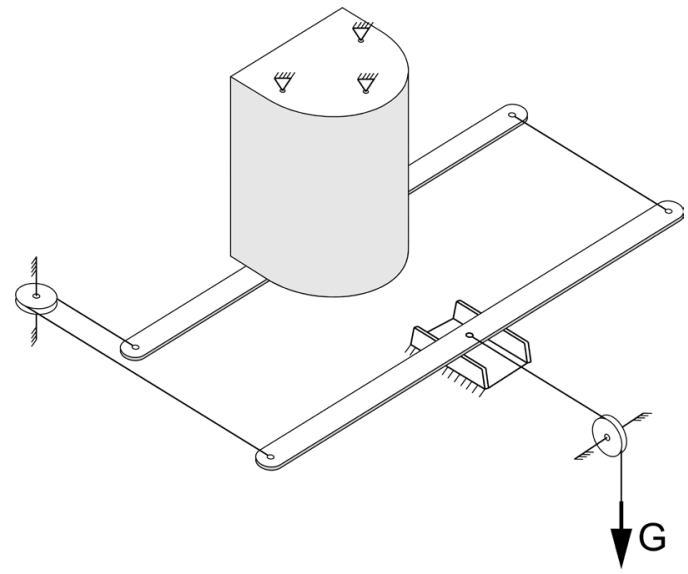

Fig. 5. Kinematic diagram of load application system

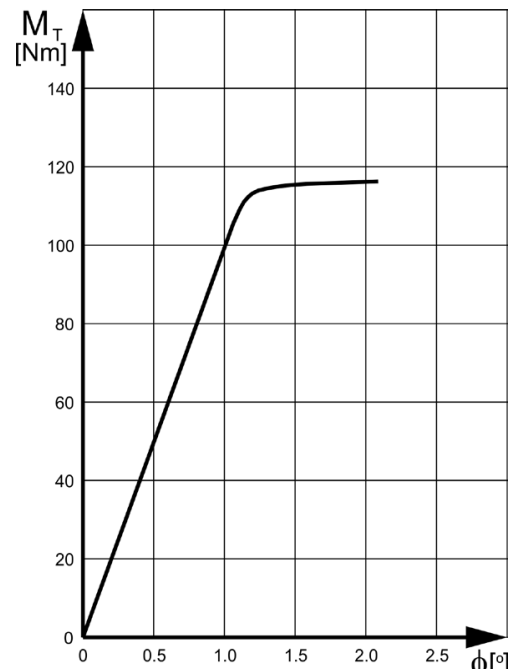

Fig. 6. Representative equilibrium path
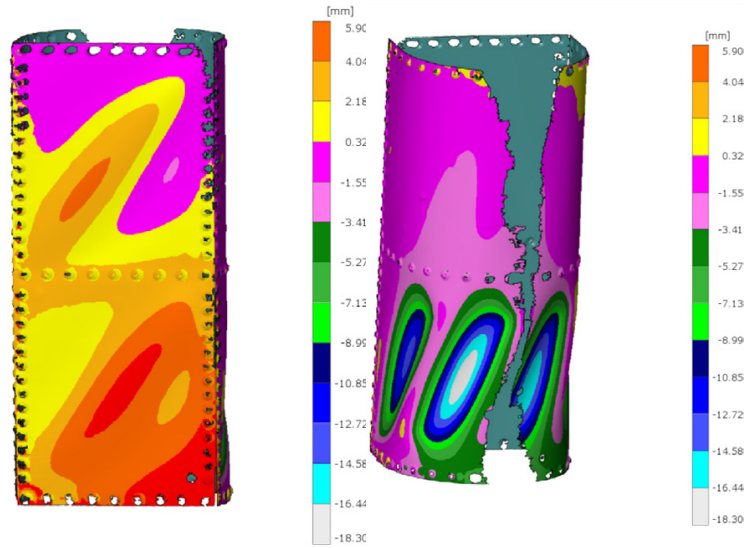

Fig. 7. Displacements distribution representation by optical scanner

It should be emphasised that the presented model material characteristic was determined in static stress conditions. In the case of rapid increases of stress level reflecting e.g. sudden changes duringcovering buckling, there is a risk of damage or destruction of the model. Therefore the presented experimental stand was equipped in a system preventing sudden change of distance between ribs.

High optical sensitivity of the polycarbonate enabled using photoelasticity methods. As a result, distribution of optical effects was achieved, which may be interpreted as proportional to effective stress distribution (Fig. 8).

Notably, the model post-critical deformations are non-symmetrical. In the cylinder part, only 

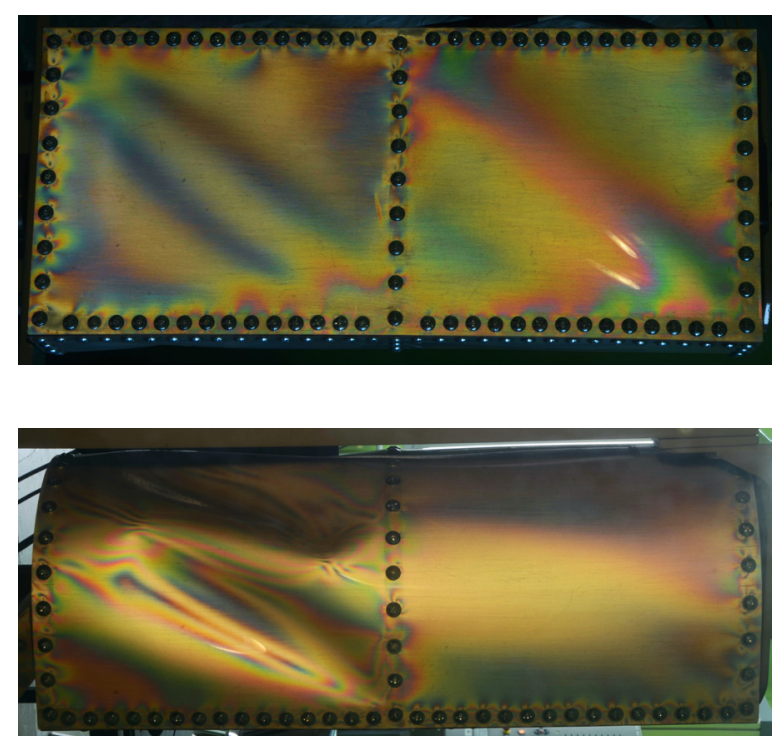

Fig. 8. Optical effects distribution in polarised light

the lower section of the covering underwent stability loss, while in the flat area, corresponding to a wing sparwall, folds of the lower segment were deeper and had different from than in the case of the upper segment. This is caused by the asymmetry of the boundary conditions, which in major degree decide on the behaviour of nonlinear system. Despite identical thickness of upper and lower rib, the rigidity of the first one is increased by the system of mounting to the stand.

\section{NUMERICAL ANALYSIS}

Results obtained during the experiment enable qualitative and to a certain degree quantitative analysis of displacements distribution (quantitative analysis is dependent on measurements preciseness, type of used optical scanner, and number of measuring points). In the case of the presented methods, quantitative measurements corresponded solely to representative values, and therefore it was not possible to precisely determine reduced stress distribution in the area of the whole covering.

Even though the experiment itself was simplified, its results were a sufficient basis for creation of an adequate numerical model.

The model was created using MSC PATRAN software, based on the finite elements method. Nonlinear numerical analyses were conducted using MSC MARC software.

The objective of the further numerical tests was to obtain a satisfying representation of both covering deformation character and representative equilibrium path.After achieving the required convergences of results of the numerical calculations and the experiment, numerically determined effective stress distribution may be considered credibleon the basis of solutions univocityrule [4]. According to the rule, one and only one stress state may match a specific structure deformation state.

Distribution of optical effects in the polarised light, on the other hand, enables aqualitative verification of stress distribution received numerically [10].

The nature of nonlinear numerical analyses is that the task realisation is carried out in stages. In each stage, a prognostic procedure is carried out, the purpose of which is to determine next variant of relation between state parameters set and control parameters corresponding to a static equilibrium location. The relation is a system equilibrium path and equals hypersurface in state hyperspace $[2,3]$. It is a correlation realising the matrix equation of residual forces $[9,14]$ :

$$
\mathrm{r}(\mathrm{u}, \Delta)=0
$$

Where: $r$ is a residual vector, containing unbalanced force components occurring in the current system deformation state. On the other hand, $u$ is a state vector, corresponding to structure nodes displacement components in the current geometrical configuration. Matrix $\Delta$ contains control parameters corresponding to the current load. Control parameters set may be expresses by single parameter $\lambda$, which is a load system function. The equation (1) assumes then the following form:

$$
\mathrm{r}(\mathrm{u}, \lambda)=0
$$

called monoparametric equation of residual forces.

Each of the phases of nonlinear procedure usually contains also a corrective phase, based on realising by the system an additional equation called increase control equation [3]:

$$
\mathrm{c}\left(\Delta \mathrm{u}_{\mathrm{n}}, \Delta \lambda_{\mathrm{n}}\right)=0
$$

where increases:

$$
\Delta u_{n}=u_{n+1}-u_{n} ; \quad \Delta \lambda_{n}=\lambda_{n+1}-\lambda_{n}
$$

correspond to the transfer from state nto state $n+1$.

In numerical representations of coverings post-critical deformations, it frequently becomes necessary to use corrective strategies based on the "arc length control", which includes methods of Rixand Crisfield [2, 3]. In the case of the issue presented, the most ef- 

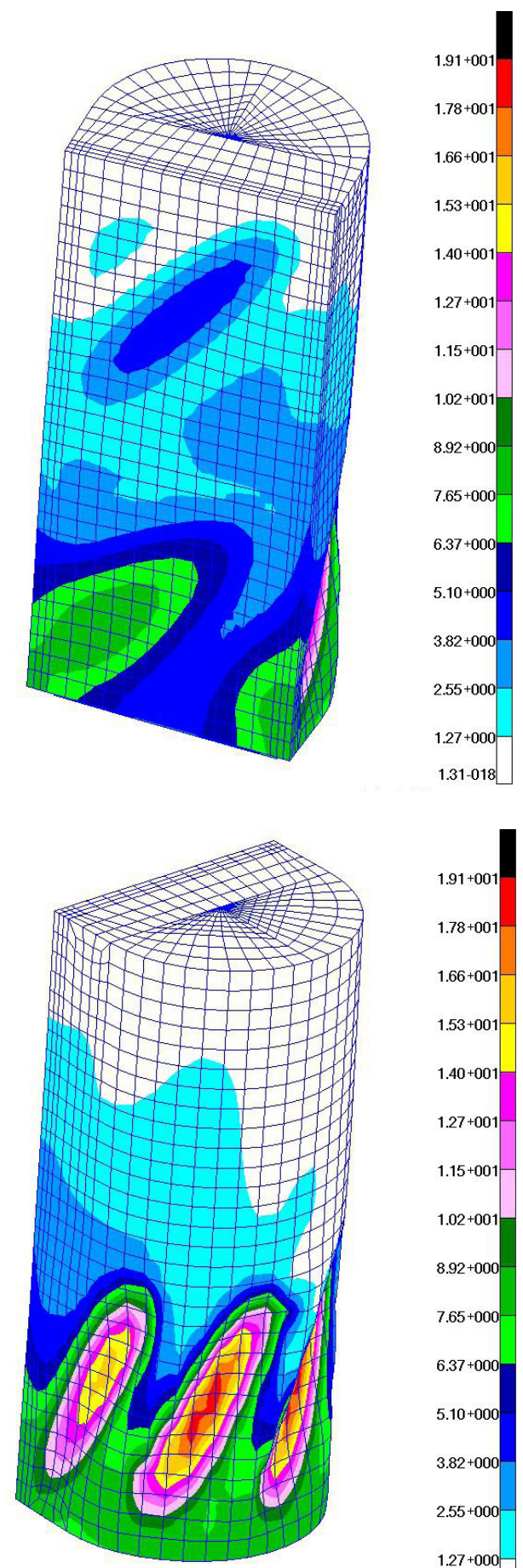

Fig. 9. Displacements distribution received as a result of numerical analysis [mm]

fective is a corrective strategy based on stress control, consisting of iterative selection of state parameters with maintaining constant value of the control parameter [9].

Numerical model was based on a meshof finite elements consisting of 2439 bilinear plateshell elements and 2604 nodes.

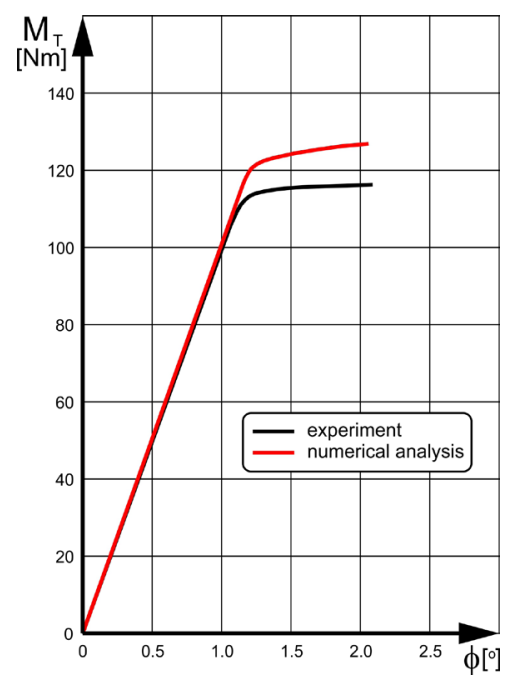

Fig. 10. Comparison of representative equilibrium paths

As a result of the analysis, displacements distribution was received (Fig. 9), on the basis of which the representative equilibrium path was determined (Fig. 10).

Comparison of results of the calculations and the experiment allows to assess similarity of displacements distribution as sufficient, both in the qualitative and quantitative aspect. In the flat part of the model, deformation took form of single fold in the upper segment and a deeper double fold in the lower segment. Torsion boxcovering underwent post-critical deformation only in the lower segment. Named features of the deformation observed during the experiment were represented as a result of nonlinear numerical analysis, which confirms correctness of utilised finite elements mesh, as well as of the assumed boundary conditions.

Representative equilibrium paths show satisfying consistence as well, even though the numerical model, due to the idealised geometry, characterizes with slightly heightened rigidity in the area of post-critical stress.

Observations above allow to accept the credibility of numerically determined effective stress distribution (Fig. 11), the comparison of which with the optical effects distribution received during the experiment allows to observe clear qualitative similarity.

It should be emphasised as well that nonlinear numerical analysis was conducted on a basis of model characterising with relatively low density of mesh of finite elements. Attempts to increase its density led to appearance of incorrect forms of post-critical deformations. 

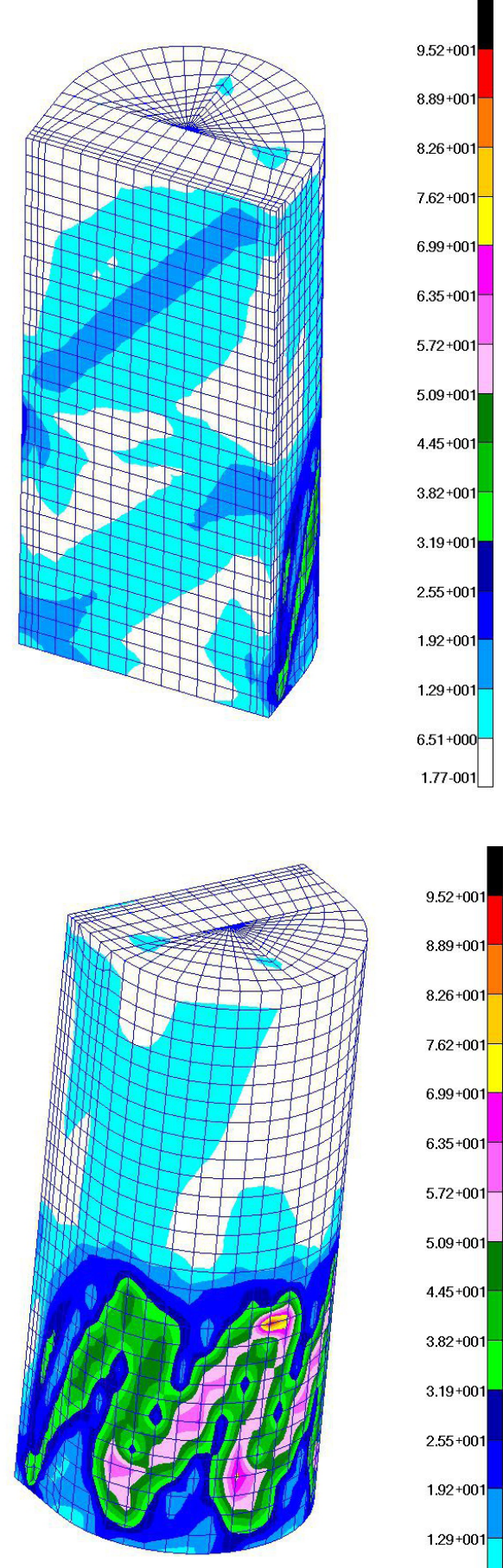

Fig. 11. Reduced stress distribution acc. to the Tresca hypothesis [Mpa]

\section{CONCLUSIONS}

Solutions presented above prove usefulness of determining numerically stress distribution in thin-walled elements of aircraft structures in conditions of post-critical stress. However, it should be emphasised that even small change in correct representation of boundary conditions and of proportions betweenrigidities of model parts may lead to receiving incorrect results. Due to the ideal character of geometric features of numerical representation and consistency of connections used in it, it is necessary to perform small, adequate corrections of coverings thickness allowing to achieve correct behaviour of the whole structure.

An additional factor frequently causing failures in the area of representation of post-critical deformations of aircraft structures systems is incorrect density offinite elements mesh. Differently than in the case of linear analyses, attempting to increase a number of elements results in not only significant increase of task size, but also causes distortions of results generated by complex nonlinear algorithms and equally complex procedures of selection of correct branchesof equilibrium pathsin bifurcation points. Too small number of finite elements, on the other hand, does not allow to adequatelyrepresent rigidity of the object components, also leading to incorrect results of the analyses.

As a result, a necessary condition of accepting credibility of nonlinear numerical analyses results is their experimental verification. It should be noted that a basis of such verification may be relatively simple and cheap model experiment using materials allowing to determine features of the tested object at relatively low loads values.

A drawback of the polycarbonate used in the described experiment as model material is mostly lack of availability of semi-manufactured plate components of small width, allowing their use in models of small size. It significantly complicates the preparation process of experimental model itself as well as of the experimental stand.

Presented methodology of obtaining stress distributions in analysed load-bearing structure in conditions of post-critical deformations, also allows to obtain the displacements level causing possible destruction of the torsion box. It enables to apply a relevant protection system, preventing excessive deformations, like internal space truss or spacer, limiting the distance between forming ribs.

\section{FURTHER RESEARCH}

Despite more and more frequent usage of composites in aircraft structures, there also appears a trend of using polymer materials, particularly in the case of unmannedaircraft structures. It is caused first and foremost by increasing possibilities of $3 \mathrm{D}$ printing and by relatively 
low costs of components structures production by using this technology.

Considering the rapid development of the aforementioned devices, perfecting features of materials used in the 3D printing, and possibility of generating structural elements of increasingly large dimensions, it should be assumed that both for technical and economic reasons, the printed components will soon be commonly used also in crew, and even airliner aircrafts.

Therefore, it seems justified to conduct research on all variants of thin-walled printed elements of aircraft structures.

In reference to notes presented above, it is planned to use the experimental stand described in the paper foe experiments using printed models, as well as to use the described methods of determining the stress distribution by verified experimentally nonlinear numerical analyses.

\section{REFERENCES}

1. Arborcz J, Post-buckling behavior of structures. Numerical techniques for more complicated structures. Lecture Notes In Physics 1985; 288: 83-142.

2. Bathe K. J. Finite element procedures. Prentice Hall, 1996.

3. Crisfield MA, Non-linear finite element analysis of solid and structures, J. Wiley \& Sons, 1997.

4. Doyle JF, Nonlinear analysis of thin-walled structures, Springer-Verlag,2001.

5. Dobrzański P, Czarnocki P, Lorenz Z, Shell structures - theory and application, CRC Press, 2013.

6. Debski H, Teter A, Kubiak T: Numerical and experimental studies of compressed composite columns, Composite Structures 2014;118:28-36.

7. Dębski H., Kubiak T., Teter A. Experimental investigation of channel-section composite profiles' be- havior with various sequences of plies subjected to static compression. Thin-Walled Structures, 2013; 71: 147-154.

8. Dębski H. Experimental investigation of postbuckling behavior of composite column with top-hat cross-section. Eksploatacja i Niezawodnosc — Maintenance and Reliability, 2013; 16 (2): 1056-109.

9. Felippa CA, Crivelli LA, Haugen B, A survey of the core-congruential formulation for nonlinear finite element, Archive of Computer Methods in Engineering 1994.

10. Kopecki T, Numerical-experimental analysis of the post-buckling state of a multi-segment multimember thin-walled structure subjected to torsion, Journal of theoretical and applied mechanics 2011; 49(1), 227-242

11. Kopecki T, Bakunowicz J, Lis T, Post-critical deformation states of composite thin-walled aircraft load-bearing structures, Journal of Theoretical and Applied Mechacnics 2016;54(1), 195-204.

12. Lynch C. A. Finite element study of the post buckling behavior of a typical aircraft fuselage panel, PhD Thesis, Queen's University Belfast, 2000.

13. Kopecki T., Mazurek P. Problems of numerical bifurcation reproducing in post-critical deformation states of aircraft structures. Journal of Theoretical and Applied Mechanics, 2013; 51(4): 969-977.

14. Riks E, An incremental approach to the solution of snapping and buckling problems, International Journal of Solid and Structures 1979; 15: 529-551.

15. Rudawska A., Dębski H. Experimental and numerical analysis of adhesively bonded aluminium alloy sheets joints, EksploatacjaiNiezawodnosc - Maintenance and Reliability, 2011; 49(1): 4-10.

16. Yeh M., Lin M., Wu W. Bending buckling of an elastoplastic cylindrical shell with a cutout Engineering Structures, 1999; 21(11): 996-1005. 